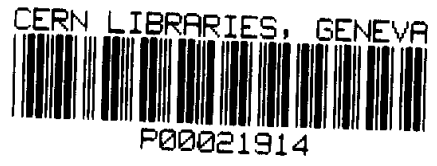

\title{
A VME based imaging system for the Virgo Project
}

A. Bazan, R.Flaminio*, P. Kramer, J.C. Lacotte, J.Lecoq, L. Massonnet, B. Mours, R. Morand, S. Tissot, D. Verkindt, M. Yvert

$\angle A P P, B P 110$

74941 Annecy le Vieux CEDEX

France

\begin{abstract}
The component test as well as the setting up of the large interferometer of the Virgo project requires accurate monitoring of the laser beam position and shape. Such a monitoring should work in real time and should be well integrated to the interferometer control system. In this paper we present a beam imaging system based on a CCD camera interfaced to VME and graphic analysis tools specially developed for this application.
\end{abstract}

(Submitted to Astroparticle Physics)

*INFN, sez. di Pisa, via Livournese 582/A, 56010 San Piero a Grado, Pisa, Italy 



\section{Introduction}

The Virgo detector[1] is a large ( $3 \mathrm{~km}$ armlength) Michelson interferometer designed for the detection of gravitational waves. The experiment aims for the measurement of the arm length variations induced by gravitational waves produced by astronomical sources. Its design sensitivity is $\Delta \mathrm{l}=10^{-18} \mathrm{mHz}^{-1 / 2}$ around $100 \mathrm{~Hz}$. To reach such a sensitivity, one makes use of ultra stabilized high power YAG laser, light recycling technics, Fabry Perot cavities in the interferometer arms, very low losses mirrors and fairly sophisticated seismic isolation systems. Under normal operation, the interferometer stays locked on its optimum tuning 24 hours over a day. To achieve such a requirement, it is mandatory to set up the essential components of the interferometer with an accurate control and monitoring of the laser beam position and shape.

A wide selection of commercial products is available on the market, but none of them matches our speed, dynamic, flexibility, and resolution specifications. Therefore a custom beam imaging system has been developed. Implemented for the component setting up, its real time display and image processing will be key parts of the final automatic alignment system.

\section{The imaging system organization}

One of the major request on our imaging system is its compatibility with the interferometer control system. This system will utilize its images for monitoring. It will also analyze them to provide signals for the feedback loops. Given the size of the experiment, this control is implemented in a distributed processing scheme with Unix workstations. Its processors are located in VME crates interconnected with real time data links and computer network. Therefore we choose for the imaging system the following architecture (see figure 1.) :

- the image sensor is a CCD camera which produces an analog signal.

- a VME board provides the camera synchronisation sequence, digitizes its signals and stores them in memory.

- a real time program acts as an image server. It is run by a VME based CPU or by a workstation connected to the VME bus.

- a program running on a workstation acts as a client and displays the images with various options chosen by the user with a graphic interface.

- an optional video monitor can be connected to the VME camera interface.

\subsection{The image sensor}

A commercial Reticon M9256 camera [2] is used. Its image sensor is a matrix of 256 by 256 square $40 \mu \mathrm{m}$ photodiodes pixels which cover a total active area of about $1 \mathrm{~cm}^{2}$. Compared to conventional video cameras this camera has several advantages : a higher number of frames per second, a non interlaced format, discrete photo sensing elements, squared pixels. Its housing provides a good mechanical stability and its sensor is aligned with respect to registration holes. Its electronics includes a preamplifier. It is driven by an external clock provided by the VME camera interface board and its analog output signal has a \pm 2 Volts range and a signal over noise dynamic larger than 1000 .

This image sensor has a quantum efficiency of only few percents for the $1.06 \mu \mathrm{m}$ wave length light produced by our NdYag laser. This is enough for our applications. 


\subsection{The VME camera interface}

The purpose of the VME interface is to digitize the camera video signal and to store the picture. The designed VME board provides the camera power supply, generates the clocking sequence, digitizes the video signal using its clock with a 10 bits flash ADC (TDC 1020 from TRW) running at $4 \mathrm{MHz}$. With such a synchronization one measures exactly the light power seen by a given pixel. This is a key feature for an absolute position measurement.

The digitized picture is stored in two memories of $32 \mathrm{~K}$ words of 16 bits, one for the odd pixels and one for the even pixels. These memories hold a full image updated at a rate of $50 \mathrm{~Hz}$ during standard operations. A special running mode allows for the capture of a single image. At any time the memories can be randomly accessed via the VME bus through a specialized register, without interference with ADC conversion and memory storage. Two words of 16 bits (i.e. two pixel contents) are read in one VME cycle. After each read cycle, the read address is automatically incremented.

\subsection{The image server}

The image server is a program running on a computer with access to the VME bus. It is activated by either a VME based CPU or a workstation connected to the VME bus. It drives the camera interface, and reads it's memory according to the request of the program running the image display. The dialogue between the image display and the image server uses the client server model with the TCP/IP protocol. Several image displays can be connected to the same image server. Therefore the image from one camera can be displayed on different sites with different options. This is one of the key feature of this system which is easily expandable to the control of several beams with cameras and image servers located in different buildings.

\subsection{The image display}

Pictures are displayed with a program running on a workstation with graphic capabilities. The program gets its images from the image server, it corrects it for hardware distorsions (pedestal, relative gain) and displays it on the screen of the workstation. The user interface is menu driven and provides various options for display and processing. One usefull option is its zoom capability. With this option, the user can select a part of the main picture for display and processing: only the selected part of the image is read by the server into the camera interface memories and the statistical computations are thus limited to this restricted area. This feature reduces the amount of data transfered on the VME bus and on the computer network.

The refresh rate is mainly a function of the graphic capability of the workstation and of the display options. When running on a SUN IPX workstation we get a typical image update rate of one per second. The processing time can be much smaller if we limit our interest to a small area of the image (zoom).

The image display has also an archiving capability. Images can be read back for further study or for processing by any other program.

In a minimal set up, using a workstation with access to the VME bus, the image server and the image display programs can be run on the same workstation. The image display program is written in C language, like the image server and uses Motif as graphic interface.

\section{Beam control}

A prototype version of this system is in use since two years ago. It is now commonly used in different laboratories for Virgo R\&D. As an example, figure 2. shows an image taken at the output of a completely misaligned triangular Fabry-Perot cavity. The spots are 
produced by the beams coming out of the cavity after an increasing number of reflection on its mirrors. The use of the logarithmic scale option provides the adequate dynamic for the visualisation of various spot sizes over a wide range of intensities. Such a feature improves the conditions of the alignment of the optical components .

\section{Position resolution}

This device can also be utilize to perform beam position measurements. The beam position $\mathrm{x}_{\mathrm{i}}$ on pixel row $\mathrm{i}$ is given by the weighted average :

$$
<x_{i}>=\frac{\sum_{j=1}^{n} x_{i j} a_{i j}}{\sum_{j=1}^{n} a_{i j}}
$$

where $\mathrm{x}_{\mathrm{ij}}$ and $\mathrm{a}_{\mathrm{ij}}$ are the positions and ADC counts of pixel $\mathrm{i}, \mathrm{j}$ and $\mathrm{n}$ the number of pixels in the considered row. If the noise per pixel is characterized by $\sigma_{\mathrm{a}}$ and its position resolution by $\sigma_{\mathrm{X}}$ one defines the standard deviation of the mean beam position as:

$$
\sigma^{2}<x_{i}>=\frac{\sigma^{2} a}{\left(\sum_{j=1}^{n} a_{i j}\right)^{2}} \sum_{j=1}^{n} x_{i j}{ }^{2}+\frac{\sigma^{2} x}{\left(\sum_{j=1}^{n} a_{i j}\right)^{2}} \sum_{j=1}^{n} a_{i j}{ }^{2}
$$

Given the stability of the pixel matrix geometry $\sigma_{X}=0$. With a gaussian beam distribution centered on the measuring row, one gets:

$$
\sigma_{<x_{i}>}=\sigma_{a} \frac{\sqrt{\sum_{j=1}^{n} x_{i}{ }^{2}}}{\sum_{j=1}^{n} a_{i j}} \approx \frac{\sigma_{a} \Delta_{x}}{\sum_{j=1}^{n} a_{i j}} \sqrt{\frac{n(n / 2+1)(n+1)}{6}}
$$

From the above relation we see that for a given cell size $\Delta \mathrm{x}$ the best sensitivity is achieved with the smallest amount of hit pixels n, and the greatest amount of ADC counts $\mathrm{a}_{\mathrm{ij}}$. A good solution is obtained with a window covering the beam extension. To maximize the ADC counts $a_{i j}$, we set the beam power to explore the full dynamic $d$ of the ADC, then

$$
\Sigma \mathrm{a}_{\mathrm{ij}} \approx \frac{\mathrm{dn}}{2}
$$

Finally, accounting for the other dimension of the beam, we perform $n / 2$ other measurements (the measurement close to the window edge have a poor resolution) and we get another factor $\mathrm{n} / 2$ on $\sigma^{2}<\mathrm{x}_{i}>$. For $\mathrm{n}$ large, on writes then:

$$
\sigma_{<\mathrm{x}>} \approx \frac{\sigma_{\mathrm{a}} \Delta_{\mathrm{x}}}{\mathrm{d}}
$$


It is worth noticing that this resolution is independent of the beam or window size. This is true if the electronics and ADC noises are the dominant ones. On the contrary, a position measurement performed with quadrant photodiodes relies much more on beam and window sizes. With a camera, the number of cells concerned by the measurement increases with the beam size. This feature is equivalent to an improvement of the ADC resolution by grouping the cells in four quadrants.

In order to measure the real position resolution of the system we first measure the electronics noise. This is done by histogramming the ADC values of the 65536 pixels of one 'dark' image after pedestal subtraction. Figure 3 shows this histogram with the results of a gaussian fit. We get:

$$
\sigma_{\mathrm{a}}=1.1 \text { ADC count }
$$

With this number, a 10 bits ADC and a pixel size of $40 \mu \mathrm{m}$, we expect:

$$
\sigma_{<\mathrm{x}>}=4 \cdot 10^{-8} \mathrm{~m} \quad \text { (per image) }
$$

Since the camera produces 50 images per second the position resolution translates to:

$$
\sigma_{<\mathrm{x}>}=.610^{-8} \mathrm{~m} / \sqrt{\mathrm{Hz}}
$$

To measure the position resolution one simulates a small beam hitting the camera with a diaphragm of about $500 \mu \mathrm{m}$ put in front of it. This diaphragm was rigidly attached to the camera to suppress any motion between camera and diaphragm. Figure 4 shows the spectral density of the positions measured along the two directions. The measured resolution is in the range $10^{-7}-10^{-8} \mathrm{~m} / \sqrt{\mathrm{Hz}}$. At low frequency the $\mathrm{x}$ resolution is dominated by the mechanical movements of the camera (thermal effect). At 'high' frequency, it is compatible with the expected value if one accounts the fact that the test beam used for this measurement did not completely saturate the dynamic of the camera. The resolution in the y a: $\quad \therefore$ is not recch the expected value because it corresponds to the low rate of pixel reading and therefore coherent low frequency electronic noises limit the resolution.

The achieved position resolution of a few $10^{-8} \mathrm{~m} / \sqrt{\mathrm{Hz}}$ is available over a dynamic range of $10^{-2} \mathrm{~m}$. The requested incident laser power is in the $\mu \mathrm{Watt}$ range. Of course, the intrinsic position resolution is worse than the one obtained with a quadrant photodiodelimited by the shot noise. Nonetheless it provides a very good stability in time within a large dynamical range because it uses small pixels well located in space and read by the same ADC.

\section{Conclusion}

The beam imaging system developed for the Virgo project and presented in this paper is already in use and provides daily help. Its architecture is well adapted to the multi processor environment of the Virgo control. It is still under development, especially for its software aspects. Its hardware will be upgraded with a private bus to connect the camera interface to the image server CPU.

\section{References}

[1] Proposal for the construction of a large interferometric detector of gravitational waves, January 1990, and Final conceptual Design, 1992, The VIRGO collaboration, available on request.

[2] Solid State Camera Products, EG\&G Reticon. 


\section{Figure Captions}

Figure 1. The components of the beam imaging system.

Figure 2. A picture presented by the image display with the main menus, the general view, a $y$ projection and a $x$ slice. Colors are used on the screen of a workstation to enhance the presentation. The image shown is the beam transmitted by completely misaligned a triangular Fabry-Perot cavity.

Figure 3. Distribution of ADC couni measured on the 65536 pixels of one image. The pedestal value is subtracted pixel by pixel The curve is the result of a fit with a gaussian distribution which gives an r.m.s. value of $1.1 \mathrm{ADC}$ count $\left(\sigma_{\mathrm{a}}\right)$.

Figure 4. Spectral density noise on the position measurement along the $\mathrm{x}$ (a) and $\mathrm{y}$ (b) axis. 


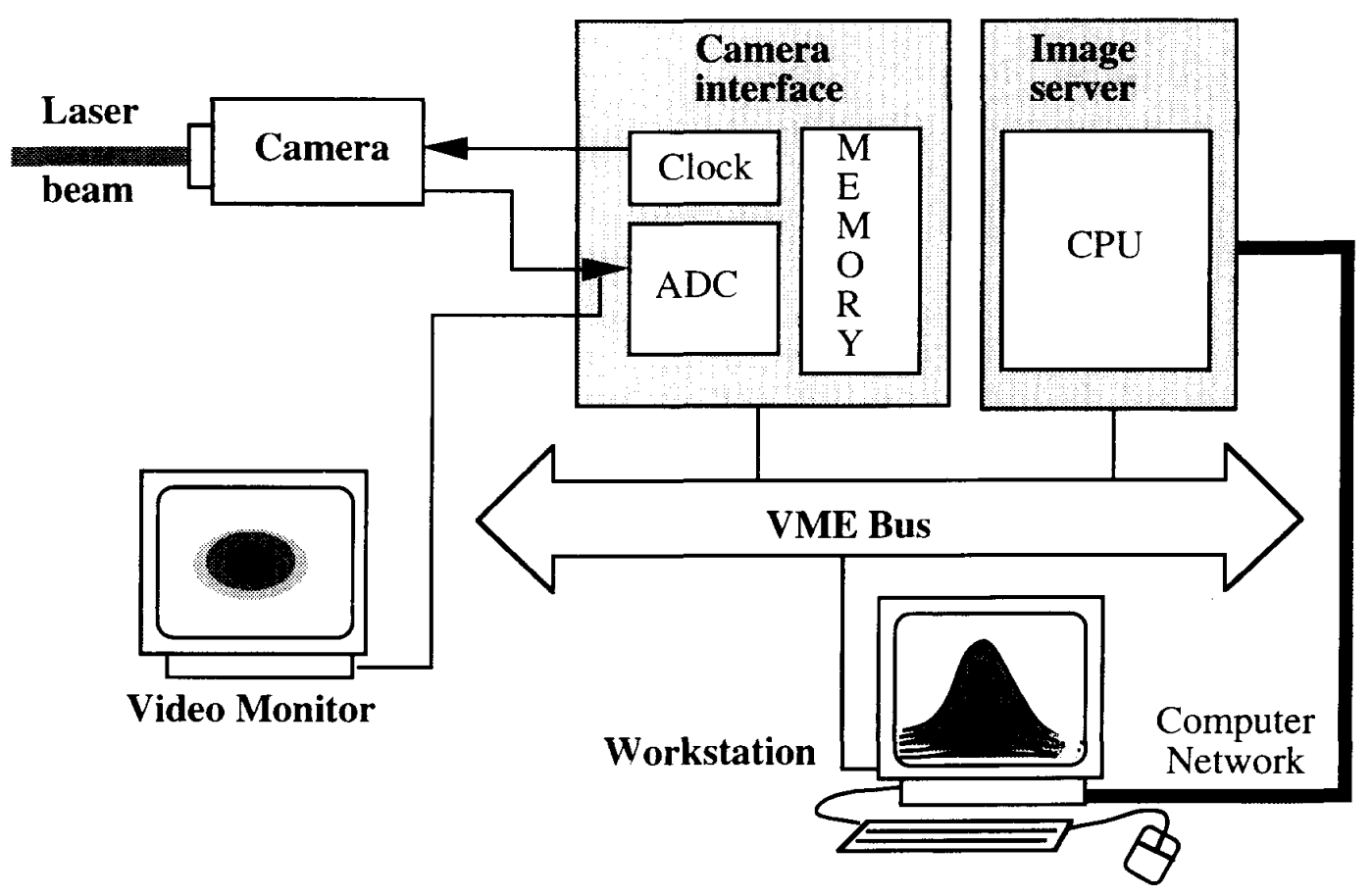

Figure 1. 


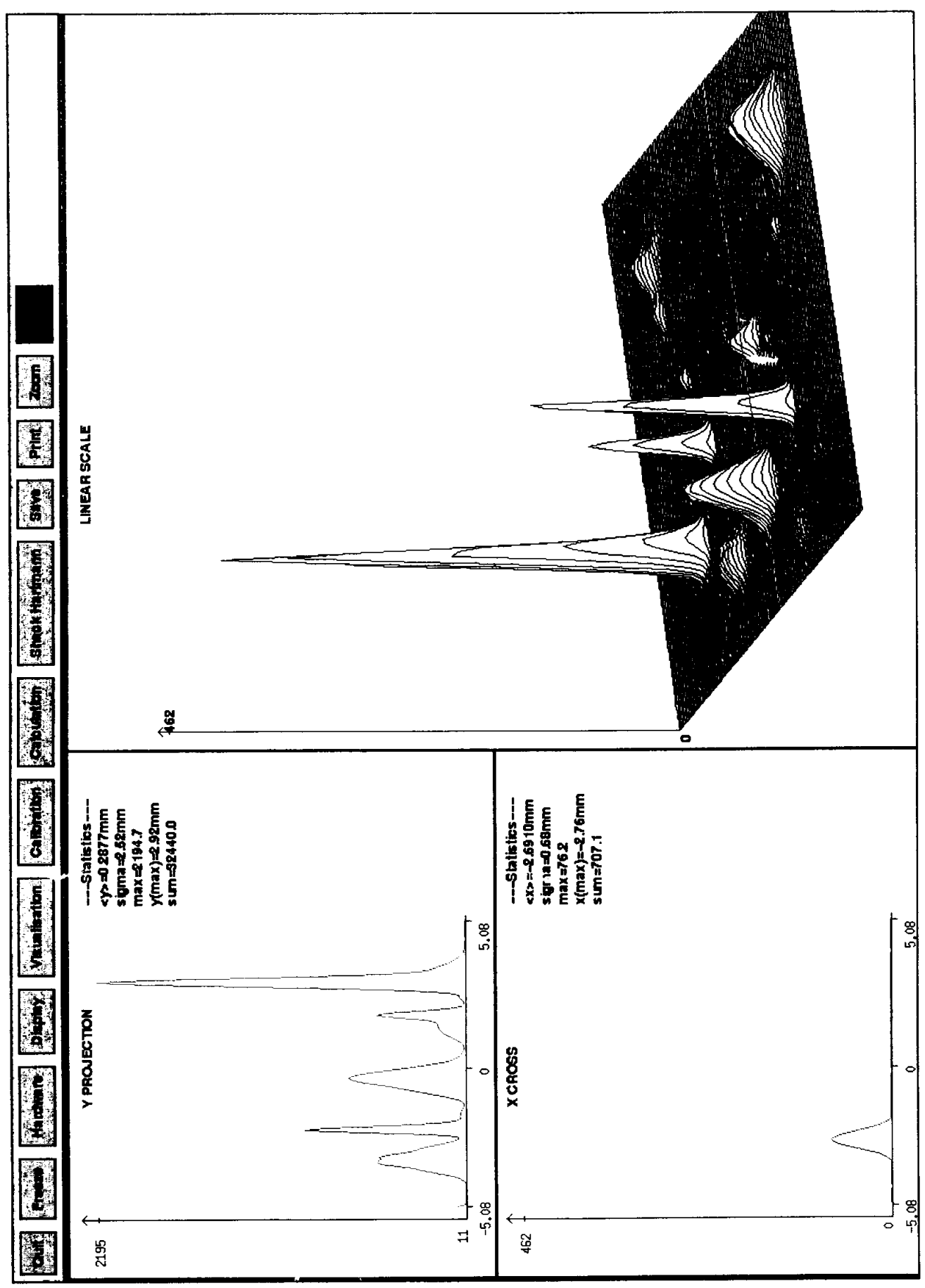

$N$
0
4
5
0
-1
$[1$ 


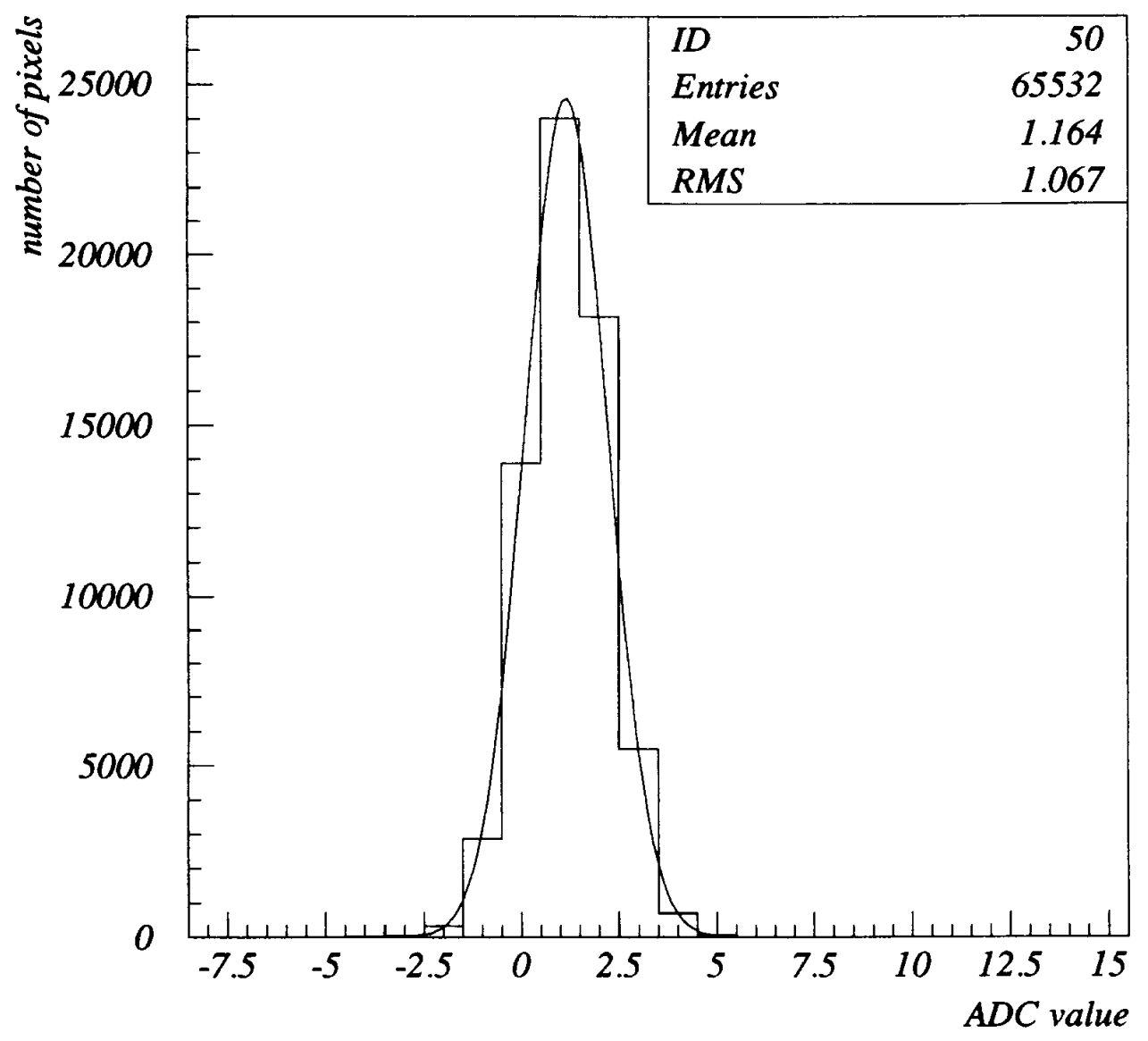

Figure 3 

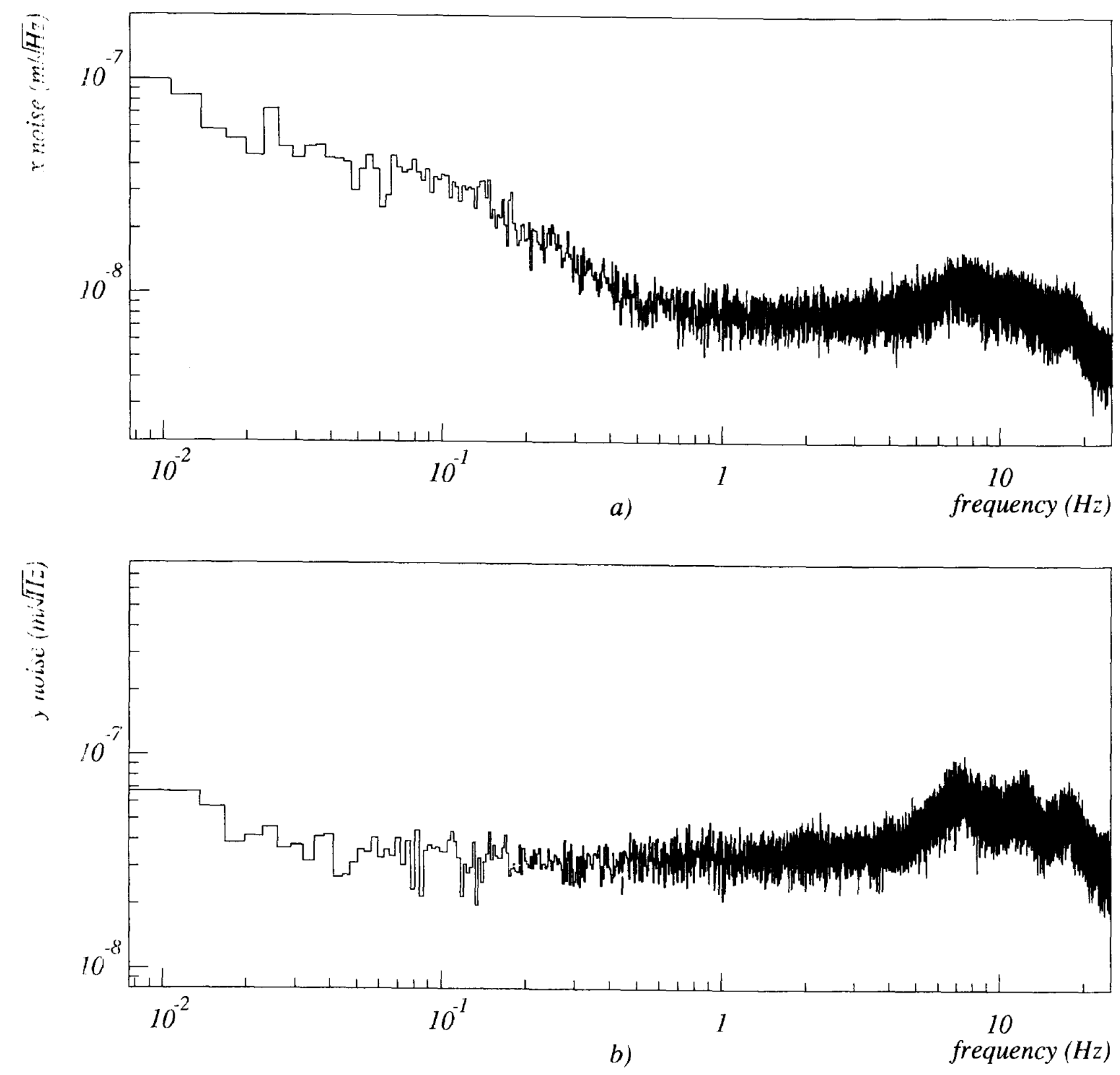

Figure 4 